are associated with six $\mathrm{O}$ atoms in a distorted octahedral arrangement when $\mathrm{Tl} / \mathrm{Pb}(1)$ and $\mathrm{O}(4)$ are on their ideal sites. However, the existence of correlated atomic displacements within the thallium(lead)-oxygen sheets is suggested by the apparent disordered arrangement in the average tetragonal structure. When shifted off of these sites (Table 2), intralayer $\mathrm{Tl} / \mathrm{Pb}-\mathrm{O}$ distances can range from 2.23 to $3.19 \AA$ whereas two short $\mathrm{Tl} / \mathrm{Pb}-\mathrm{O}$ distances remain above and below $\mathrm{Tl} / \mathrm{Pb}$. Analogous situations are found in all of the thallium-containing copper-oxide superconductors. The atomic displacements give rise to local chains or pairs of $\mathrm{T} 1 / \mathrm{Pb}$ and $\mathrm{O}$ atoms, and this could create an atomic modulation along the $a_{1}$ and $a_{2}$ axes $(14,15)$.

Two oxidation states would normally be considered for lead, $\mathrm{Pb}^{2+}$ and $\mathrm{Pb}^{4+}$. The partial replacement of $\mathrm{Tl}^{3+}$ by $\mathrm{Pb}$ in $(\mathrm{Tl}, \mathrm{Pb}) \mathrm{Sr}_{2} \mathrm{Ca}_{n-1} \mathrm{Cu}_{n} \mathrm{O}_{2 n+3}$ phases would then cause the $\mathrm{Cu}$ oxidation state to increase for $\mathrm{Pb}^{2+}$ or to decrease for $\mathrm{Pb}^{4+}$. Although some $\mathrm{Cu}^{3+}$ would remain for the $\left(\mathrm{Tl}_{0.5}^{3+} \mathrm{Pb}_{0.5}^{4+}\right) \mathrm{Sr}_{2}^{2+} \mathrm{Ca}_{2}^{2+} \mathrm{Cu}_{2.5}^{2+} \mathrm{Cu}_{0.5}^{3+} \mathrm{O}_{9}$ formulation, its concentration seems rather low for a $122 \mathrm{~K}$ superconductor. However, it is likely that the $6 s$ band, composed of $\mathrm{Tl} 6 s$, $\mathrm{Pb} 6 s$, and $\mathrm{O} 2 p$ states, crosses the Ferm level thereby creating more $\mathrm{Cu}^{3+}$ than this formulation would suggest. Work is under way to establish the actual carrier concentration in these new high $T_{\mathrm{c}}$ superconductors containing $\mathrm{Tl}$ and $\mathrm{Cu}$.

\section{REFERENCES AND NOTES}

1. Z. Z. Sheng and A. M. Hermann, Nature 332, 138 (1988).

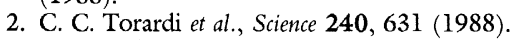

3. M. A. Subramanian et al., Nature 332, 420 (1988)

4. S. S. P. Parkin et al., Phys. Rev. Lett. 60, 2539 (1988)

5. S. S. P. Parkin et al., ibid. 61, 750 (1988)

6. M. Hervieu et al., J. Solid State Chem. 75, 212 (1988).

7. M. A. Subramanian et al., ibid., in press.

8. Z. Z. Sheng et al., in preparation.

9. T. Siegrist et al., Nature 334, 231 (1988)

10. M. A. Subramanian et al., Science 239, 1015 (1988).

11. C. C. Torardi et al., Phys. Rev. B 38, 225 (1988).

12. D. E. Cox et al., Phys. Rev. B, in press.

13. R. D. Shannon, Acta Crystallogr. A32, 751 (1976).

14. J. D. FitzGerald, Phys. Rev. Lett 60, 2797 (1988).

15. A. W. Hewat, E. A. Hewat, J. Brynestad, H. A. Mook, E. D. Specht, Physica C 152, 438 (1988).

16. We thank P. M. Kelly, C. R. Walther, R. A. Oswald, W. J. Marshall, L. Cooke, D. Groski, G. M. Hyatt, C. M. Foris, E. Boyes, D. L. Smith, I. R. Hartmann, and C. B. Michel for their assistance.

26 August 1988; accepted 16 September 1988

\title{
The Paleomagnetic Field from Equatorial Deep-Sea Sediments: Axial Symmetry and Polarity Asymmetry
}

\section{David A. Schneider ANd Dennis V. Kent}

Paleomagnetic data from 89 equatorial deep-sea sediment cores indicate that the configuration of the time-averaged geomagnetic field depends strongly on polarity state but that it remains within 1 degree of axial symmery throughout the Pliocene and Pleistocene (last 5 million years). The relative magnitude of the nondipole field was greater by almost a factor of 2 during reverse than during normal polarity intervals. These results thus support earlier suggestions that there may be a standing (nonreversing) component of the geomagnetic dynamo.

$\mathrm{T}$ HE TIME-AVERAGED GEOMAGNETIC field can deviate from the field of a geocentric axial dipole (1). Although the difference is small, amounting to a few degrees, the deviation, attributed to a longterm nondipole field (NDF), provides a means to examine the nature of the underlying dynamo.

One intriguing aspect of the NDF is its apparent dependence on polarity state. Indications of this dependence have come, for example, from Wilson's (1) examination of piston core data (2) and also from his study of paleomagnetic data from the U.S.S.R

Lamont-Doherty Geological Observatory and Department of Geological Sciences, Columbia University, Palisades, NY 10964.
(3). Also, Merrill and McElhinny (4), using a spherical harmonic analysis of global paleomagnetic data, showed that the dominant NDF terms (the axial quadrupole and axial octupole) are larger by nearly a factor of 2 for reverse than for normal polarity data. These results, although suggestive of polarity dependence, were open to alternative explanation: the limited age control allowed that differences between normal and reverse directions might result from changes in the NDF with time and be fundamentally unrelated to polarity.

We have studied these possible NDF effects in well-dated Pliocene to Pleistocene deep-sea sediment cores from equatorial latitudes (all but one within $\pm 15^{\circ}$ ). Near the equator, the axial quadrupole NDF is greatest and paleomagnetic directions close to horizontal and thus are least susceptible to spurious shallowing caused by sedimentary inclination error. Pelagic sediments are known to provide high-quality paleomagnetic data (5) and can be used to study the time-averaged properties of the field because bioturbation and the low sedimentation rates (about $1 \mathrm{~cm}$ per 1000 years) act to average secular variation.

In an earlier study of 29 piston cores from the equatorial Indian Ocean (6), we concluded that the NDF was different during the Brunhes [ 0 to $0.73 \mathrm{Ma}$ (million years ago); normal polarity] from that during the Matuyama ( 0.73 to $2.47 \mathrm{Ma}$; reverse polarity) interval. Resolution in the earlier Gauss (2.47 to $3.40 \mathrm{Ma}$; normal polarity) and Gilbert (3.40 to $5.35 \mathrm{Ma}$; reverse polarity) chrons was poor, and therefore the supposed polarity dependence could not be adequately tested. In this report, we present data from an expanded set of deep-sea sediment cores ( 60 additional cores for a total of 89) taken from the Indian, Atlantic, and Pacific oceans (Fig. 1). By studying cores distributed around the equatorial region, we could describe better the axial quadrupole field and also test whether the dipole axis was inclined to the rotation axis, that is, whether the paleomagnetic field was axially symmetric. The deep-sea sediments studied were recovered with standard piston coring techniques. Data for 29 of the 89 cores are in (6); tabulated data for the remaining cores are available from the authors (7).

Because none of the cores were oriented in azimuth, we could not determine absolute

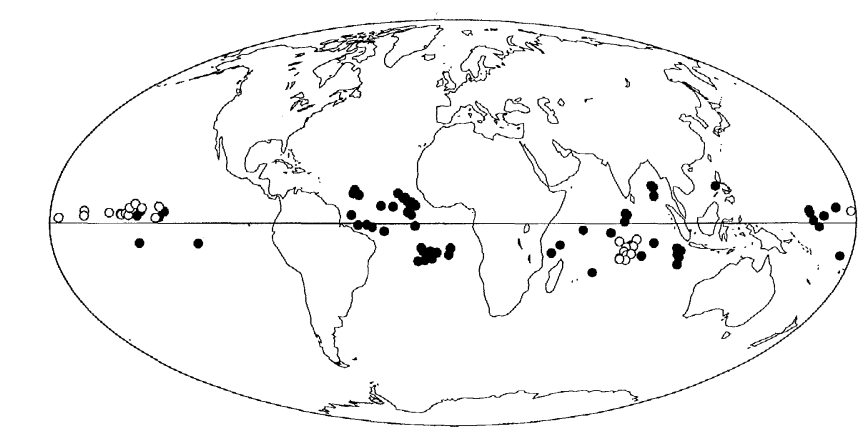

g. 1. Location of the 89 equatorial deep-sea sediment cores used in this study. Closed circles show cores containing only Brunhes or Matuyama age sediments, or both (that is, younger than $2.47 \mathrm{Ma}$ ). Open circles show cores also containing Gauss or Gilbert age sediments, or both (older than $2.47 \mathrm{Ma}$ ). 


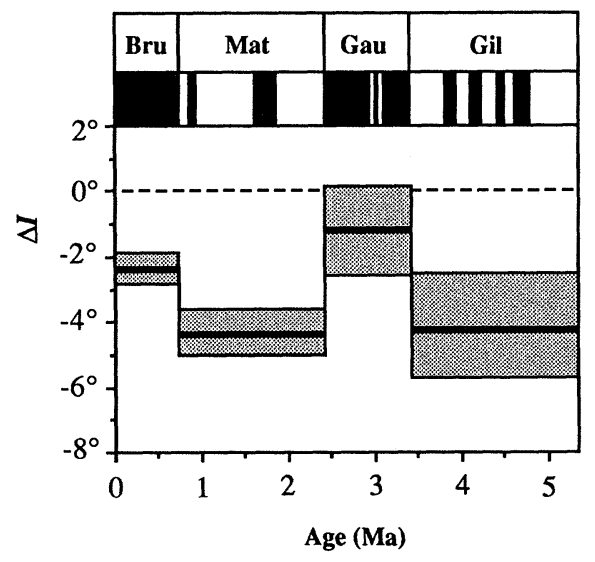

Fig. 2. Inclination anomaly $(\Delta I)$ versus age for the Brunhes (Bru), Matuyama (Mat), Gauss (Gau), and Gilbert (Gil) geomagnetic polarity chrons of the Pliocene and Pleistocene. One standard error is shown. Black indicates intervals of normal polarity, white of reversed polarity. No data corresponding to subchrons are included.

declination. We have thus used a maximum likelihood technique (8) to compute mean inclination for specified intervals in each core; the errors involved from such averaging are negligible at equatorial latitudes (6). To examine these mean inclination data easily, we have calculated inclination anomalies, $\Delta I$ 's (9):

\section{$\Delta I=$ (observed mean inclination) \\ - (geocentric axial dipole inclination)}

We inverted the sign of reverse polarity inclinations so that normal and reverse polarity data could be averaged together or directly compared. We computed the dipole inclination after determining paleopositions (at the mean age of the relevant samples) from a hotspot-based absolute plate motion model [model AMl-2 in (10)].

We calculated average $\Delta I$ 's for the four named chrons of the Pliocene and Pleistocene (Brunhes, Matuyama, Gauss, and Gilbert) to test for polarity-dependent behavior of the time-averaged geomagnetic field by averaging data from all cores for which there were six or more samples in the specified chronozone (11). We excluded all data reflecting subchrons: thus, each average included data of one polarity only.

The resultant $\Delta I$ averages (Table 1) have small (negative) magnitudes for all four polarity chrons; however, the two normal polarity (Brunhes and Gauss) averages have smaller deviations from dipole directions than do the two reverse polarity (Matuyama and Gilbert) averages (Fig. 2). The Brunhes anomaly $\left(-2.4^{\circ} \pm 0.5^{\circ}\right)$ is not appreciably different from the Gauss anomaly $\left(-1.2^{\circ} \pm 1.3^{\circ}\right)$; nor is the Matuyama anomaly $\left(-4.3^{\circ} \pm 0.7^{\circ}\right)$ different from the Gilbert anomaly $\left(-4.3^{\circ} \pm 1.8^{\circ}\right)$ (Table 1$)$. Be- cause the four chron averages show no obvious trend with time, these data may be grouped by polarity. The best estimate of normal polarity $\Delta I$ (Table 1 ) was calculated by first averaging the Brunhes mean with the Gauss mean in each core before averaging between cores. We similarly averaged the Matuyama and Gilbert data. This average differs slightly from the separated $\mathrm{Ma}$ tuyama and Gilbert results because of slight differences in how this averaging procedure weights each core value.

The polarity-dependent pattern cannot be the result of the systematic shallowing of inclination in sediments [for example (12)] which should be similar in normal and reverse polarity intervals. Some contamination of the data set with unremoved present-day field overprints might be an alternative source of error. The present-day field contains a substantial axial quadrupole $\left(g^{0}{ }_{2}\right)$ term $[-2073 \mathrm{nT}(13)]$ that adds an outward component to the field at the equator. Hence, overprinting could account for the small negative $\Delta I$ values that we calculated for the normal polarity intervals, but it would not have produced larger magnitude anomalies in the reverse polarity chrons. Thus, the systematic variation of inclination anomalies apparently reflects the actual behavior of the time-averaged geomagnetic field.

We tested whether this variation might reflect secular changes in the time-averaged field that do not depend inherently on polarity state by calculating inclination anomalies for narrow intervals in the Brunhes and Matuyama chrons ( 0 to $2.47 \mathrm{Ma}$ ), which are the better represented intervals in our data set. We assigned ages to the samples by assuming that the sedimentation rate was constant between identified magnetostratigraphic boundaries. In averaging data in the four chron groups, we had purposefully excluded all of the data from the various subchrons because the few data from these intervals might reflect the effect of transitional fields. In this calculation, however, we included inclination data from the Olduvai normal polarity subchron (1.66 to 1.88 $\mathrm{Ma})$, the only subchron that is represented by enough samples to allow a reasonable estimate of the time-averaged $\Delta I$. The within-chron $\Delta I$ 's (Fig. 3) show a polarity dependence that is consistent with that shown by the chron averages. The small variations in the Brunhes chron and in the reversed parts of the Matuyama chron are not significant. The Olduvai $\Delta I$, however, is small and positive and corresponds much more closely to the level in the normal polarity Brunhes anomaly than to the level in the adjacent parts of the Matuyama anomaly, which have reversed polarity. Thus a polarity dependence is seen at both chron and subchron scales.

Our interpretation is largely in agreement with the spherical harmonic analysis of Merrill and McElhinny (4). Although they used some of the same piston core data that we analyzed, most of the data are not shared. To compare our data with theirs, we as-

Table 1. Average $\Delta I$ for the four geomagnetic polarity chrons of the Pliocene and Pleistocene.

\begin{tabular}{lcccccc}
\hline \multicolumn{1}{c}{ Polarity/chron } & $\begin{array}{c}\text { Mean age } \\
(\mathrm{Ma})\end{array}$ & $N^{*}$ & $\begin{array}{c}\text { Lati- } \\
\text { tude } \dagger\end{array}$ & $\Delta I$ (uncorr.) $\ddagger$ & $\Delta I$ & $\begin{array}{c}g^{0}{ }_{2} / g^{0}{ }_{1} S \\
(\%)\end{array}$ \\
\hline Normal/Brunhes & 0.3 & 86 & $1.0^{\circ} \mathrm{S}$ & $-2.57 \pm 0.50$ & $-2.37 \pm 0.50$ & 2.8 \\
Reverse/Matuyama & 1.4 & 54 & $2.6^{\circ} \mathrm{S}$ & $-5.25 \pm 0.72$ & $-4.30 \pm 0.71$ & 5.0 \\
Normal/Gauss & 2.9 & 22 & $2.8^{\circ} \mathrm{S}$ & $-3.56 \pm 1.35$ & $-1.20 \pm 1.34$ & 1.4 \\
Reverse/Gilbert & 3.8 & 17 & $3.0^{\circ} \mathrm{S}$ & $-7.39 \pm 1.76$ & $-4.25 \pm 1.75$ & 5.0 \\
Normal/all & - & 89 & $1^{\circ} 3^{\circ} \mathrm{S}$ & $-2.88 \pm 0.49$ & $-2.41 \pm 0.49$ & 2.8 \\
Reverse/all & - & 54 & $2.7^{\circ} \mathrm{S}$ & $-5.35 \pm 0.74$ & $-4.13 \pm 0.72$ & 4.8 \\
\hline
\end{tabular}

*Number of cores included in average. †Mean latitude of included cores. ‡Average inclination anomaly with plate motion corrected and uncorrected (uncorr.); one standard error is shown. \$Ratio of axial dipole component to axial quadrupole component (NDF); calculated from the plate motion corrected anomaly value.

Table 2. Results of least-squares analysis for best fitting pole position (29); Lat, latitude; Lon, longitude; and CSD, circular standard deviation.

\begin{tabular}{|c|c|c|c|c|c|c|}
\hline \multirow{3}{*}{ Chron } & \multicolumn{6}{|c|}{ Best fit dipole axis } \\
\hline & \multicolumn{2}{|c|}{ Without plate motion } & \multicolumn{4}{|c|}{ With plate motion } \\
\hline & Lat $(\mathrm{N})$ & Lon $(E)$ & Lat $(\mathrm{N})$ & Lon $(E)$ & Kappa & CSD \\
\hline Brunhes & $89.5^{\circ}$ & $169.6^{\circ}$ & $89.4^{\circ}$ & $167.3^{\circ}$ & 19,700 & $0.6^{\circ}$ \\
\hline Matuyama & $89.0^{\circ}$ & $340.7^{\circ}$ & $89.4^{\circ}$ & $351.4^{\circ}$ & 11,300 & $0.8^{\circ}$ \\
\hline Gauss & $88.1^{\circ}$ & $307.1^{\circ}$ & $89.3^{\circ}$ & $201.0^{\circ}$ & 1,487 & $2.1^{\circ}$ \\
\hline Gilbert & $87.6^{\circ}$ & $344.6^{\circ}$ & $89.2^{\circ}$ & $70.6^{\circ}$ & 414 & $4.0^{\circ}$ \\
\hline
\end{tabular}




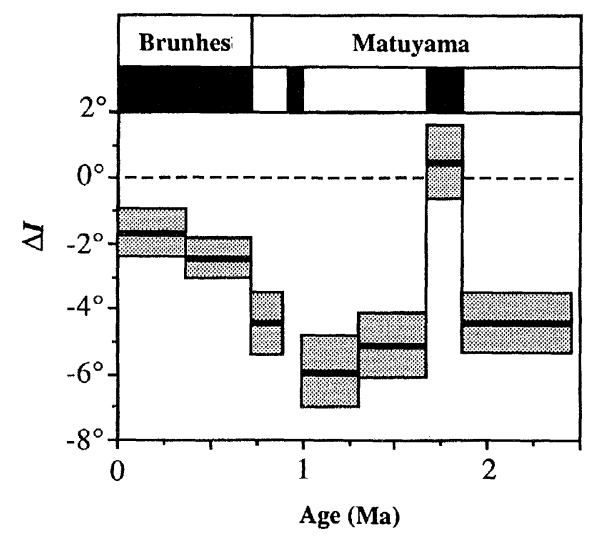

Fig. 3. Inclination anomaly $(\Delta I)$ versus age for intervals in the Brunhes (normal polarity) and Matuyama (reversed polarity) chrons with data from the Olduvai (normal polarity) subchron included.

sumed that our $\Delta I$ values reflect the axial quadrupole $\left(g_{2}^{0}\right)$ component of the NDF (Fig. 4). Although equatorial sites will be affected by the sum of all even-valued zonal terms, none of the odd-valued terms (for example, $g_{3}^{0}$ ) should contribute. The $-2.4^{\circ}$ normal polarity $\Delta I$ that we measured corresponds to a $g_{2}^{0}$ to $g_{1}^{0}$ ratio of about $3 \%$. The dipole term $\left(g_{1}^{0}\right)$ is negative for normal polarity and thus the quadrupole term $\left(g^{0}{ }_{2}\right)$ is also negative. The $-4.1^{\circ}$ reverse polarity $\Delta I$ corresponds to a ratio of about $5 \%$. Here $g^{0}{ }_{1}$ and $g_{2}^{0}$ are positive. We cannot determine from directional measurements alone whether the difference in the ratios between polarities is caused by variation in the dipole or in the quadrupole. A changing quadrupole seems most likely, however, because the required change in the time-averaged dipole intensity is too large (nearly a factor of 2) to have escaped detection in paleointensity studies [for example (14)].

Merrill and McElhinny (4) estimated that the normal and reverse polarity axial quadrupole to axial dipole ratios were $5 \%$ and $8 \%$, respectively. Our estimates of the NDF ratios are smaller; the overall magnitude of the NDF is, however, sensitive to correction for plate motion, which was not made in their analysis. When we compute $\Delta I$ values without compensating for plate motion, larger values result (Table 1). This effect occurs because many of the equatorial core sites have moved northward during the past few million years (particularly those on the Indian and Pacific plates), while apparently none have moved southward.

Studies of the time-averaged field of the past few million years have generally indicated that the field does not deviate substantially from axial symmetry $(2,4,15,16)$. However, some studies of longer intervals have indicated that significant offsets do occur between the paleomagnetic and hotspot reference frames $(17-20)$. These offsets may indicate either coherent hotspot (that is, mantle) wander or, if the hotspots are fixed with respect to the rotation axis, a substantial equatorial-dipole contribution to the time-averaged geomagnetic field.

In the aforementioned polar-wander analysis, fully oriented, land-based data were used to determine paleomagnetic pole positions. We could not calculate paleomagnetic pole positions from individual cores because we have inclination-only data. Instead, we computed a single pole position that best fits the set of core data for each chron. Cores near the equator (mean latitude $1^{\circ} \mathrm{S}$ ) provide a sensitive estimate of axis tilt: at low latitudes, $1^{\circ}$ of axis tilt results in about $2^{\circ}$ of inclination change, whereas at higher latitudes inclination changes are less.

In contrast to the axially symmetric part of the NDF (discussed above), which will affect cores similarly at all longitudes, a tilt of the dipole axis will add to the anomaly in cores located in the direction of tilt and subtract in cores from the opposite direction, leading to a sinusoidal variation of $\Delta I$ with longitude. We calculated axis tilt by finding the sinusoid that best fits $\Delta I$ data (using standard least-squares procedures): the amplitude indicates the amount with $2^{\circ}$ of anomaly amplitude for each degree of tilt; the phase shows the direction (Fig. 5).

For Brunhes age core data the amplitude of the best fitting sinusoid $\left(1.2^{\circ}\right)$ corresponds to a dipole axis that is $0.6^{\circ}$ from the hotspot axis (Table 2). The uniform component is $-2.2^{\circ}$. The calculated Matuyama pole is also $0.6^{\circ}$ from the hotspot axis but is in the opposite hemisphere from the Brunhes pole. The uniform component fit to the Matuyama reverse polarity data is $-4.1^{\circ}$. The calculated Gauss dipole axis is $3.3^{\circ}$ from the hotspot axis and the uniform component is $1.2^{\circ}$. The Gilbert data give a dipole axis that is $0.8^{\circ}$ from the hotspot axis and a uniform component of $-3.8^{\circ}$.

The values for the uniform component for the Brunhes $\left(-2.2^{\circ}\right)$ and Matuyama $\left(-4.1^{\circ}\right)$ intervals are close to the average anomalies determined in our consideration of polarity dependence $\left(-2.4^{\circ}\right.$ and $-4.3^{\circ}$, respectively) (Table 1 ). The correspondence for the Gauss and Gilbert intervals is not as close $\left(1.2^{\circ}\right.$ to $-1.2^{\circ}$ and $-3.8^{\circ}$ to $\left.-4.3^{\circ}\right)$. We attribute this discrepancy to the poor longitudinal distribution of cores representing these older two chrons (Fig. 1), which prevented the complete separation of the axially symmetric and nonsymmetric effects. We can, however, remove the effects of the axisymmetric part of the NDF for the sparser Gauss and Gilbert data by specifying its expected contribution. We assume that the contribution of the axially symmetric NDF for the Gauss (normal) and Gilbert (reverse) intervals corresponds to the uniform component determined for the Brunhes (normal) and Matuyama (reverse) data. With this method, we can compensate explicitly for polarity dependence where symmetric and nonsymmetric effects might not otherwise be readily separated. The best fitting pole positions correspond to a dipole axis that is $0.7^{\circ}$ from the present-day rotation axis for the Gauss and $0.8^{\circ}$ from the axis for the Gilbert data.

The best fitting paleomagnetic pole positions are all within $1^{\circ}$ of the pole of the hotspot reference frame and show no polarity-dependent behavior nor any obvious trend with time (Fig. 6). For the four chrons the offset between the best fitting pole and the hotspot axis is not larger than the estimated errors (Table 2), and thus these small offsets are unlikely to be significant. The Fisherian (21) mean of these four directions is $89.8^{\circ} \mathrm{N}, 124.8^{\circ} \mathrm{E}$ (Fisher precision parameter, kappa $=11,324 ; \alpha_{95}=0.9^{\circ}$ ).

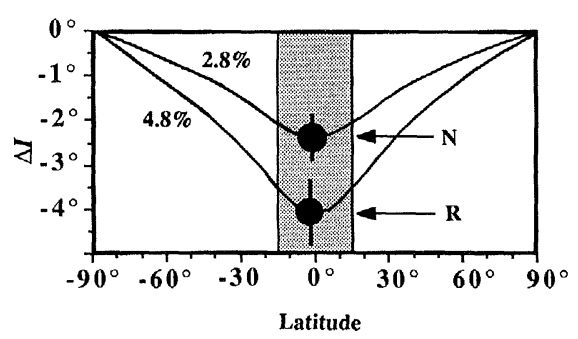

Fig. 4. Best fit normal $(\mathrm{N})$ and reverse $(\mathrm{R})$ polarity axial quadrupole effect. Axial quadrupole values are shown as percentage of the axial dipole. The inclination anomaly from an axial quadrupole field $\left(g_{2}^{0}\right)$ is given by

$$
\begin{array}{r}
\Delta I=\tan ^{-1}\left\{\frac{2 g^{0}{ }_{1} \cos \theta+g^{0}{ }_{2}\left[(9 / 2) \cos ^{2} \theta-3 / 2\right]}{g^{0}{ }_{1} \sin \theta+g^{0}{ }_{2}(3 \sin \theta \cos \theta)}\right\} \\
-\tan ^{-1}[2 \cot \theta]
\end{array}
$$

where $\theta$ is colatitude. Error bars show one standard error on $\Delta I$. Shaded portion indicates the latitude band analyzed in this study.

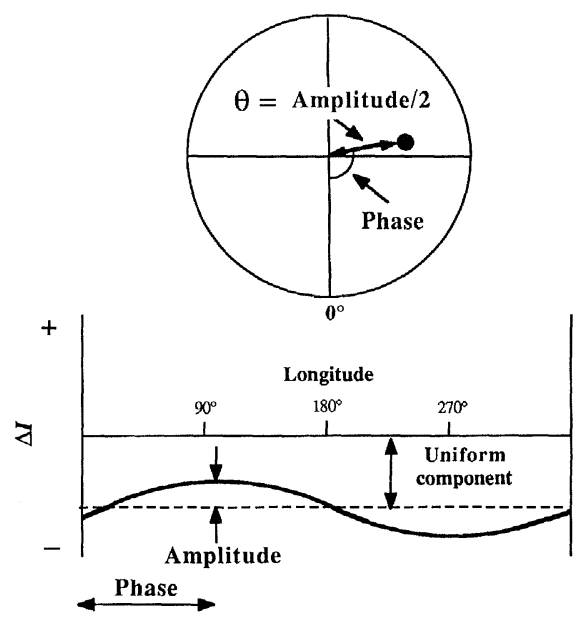

Fig. 5. Schematic illustration of the sinusoidal variation of inclination anomaly associated with a dipole tilt. 
Two mechanisms might lead to divergence between the paleomagnetic and hotspot reference frames: a long-term equatorial dipole field or hotspot wander. The youngest pole that we determined (Brunhes: nominal age $0.3 \mathrm{Ma}$ ) could not have been affected appreciably by hotspot wander. Even for rapid wander, of perhaps $1^{\circ}$ per million years $(17,18)$, the divergence between the rotation and hotspot axes would be only $0.3^{\circ}$. The near coincidence of the Brunhes pole with the hotspot axis indicates that there is no long-term equatorial dipole field and thus that the axis of symmetry of the time-averaged magnetic field corresponds, as expected, to the rotation axis. The three older poles show that the rotation and hotspot axes agree for most of the Pliocene and Pleistocene, which suggests that coherent hotspot wander must have been small or nonexistent during this interval.

Livermore et al. (16) and Opdyke and Henry (2) also showed that the best fit dipole axis was within $2^{\circ}$ of the rotation axis. Our results are, however, at odds with the analysis by Gubbins (22) of the paleomag netic compilation of Lee (23) for 0 to $5 \mathrm{Ma}$. For example, in that analysis normal polarity $\Delta I$ data from equatorial latitudes (from $10^{\circ} \mathrm{N}$ to $10^{\circ} \mathrm{S}$ ) were nearly $4^{\circ}$ more negative in the Pacific $\left(90^{\circ} \mathrm{E}\right.$ to $\left.90^{\circ} \mathrm{W}\right)$ than in the Atlantic hemisphere. This should correspond to about $2^{\circ}$ of polar offset toward the Atlantic. This apparent discrepancy between hemispheres may have been caused by the lack of plate motion correction in Gubbins's study. If we neglect the effects of plate motions in our analysis, the best fit pole is increasingly shifted toward the Atlantic hemisphere with increasing age of the interval analyzed (Table 2). The offset is caused by the more rapid northward movement of the Pacific plate compared to the equatorial Atlantic plates.

In contrast to several earlier polar-wander analyses (17-19), which included plate-motion correction, our analysis shows that the geomagnetic and hotspot reference frames remained aligned during the last $\mathbf{5}$ million years. Indications of recent episodes of rapid hotspot wander may be an artifact of the influence of the axisymmetric NDF. Unless data sites are distributed uniformly in longitude, NDF effects will tend to displace the mean paleomagnetic pole from its true position, and this effect may be confused with polar wander (24).

We have not considered our data adequate to test whether higher order, nonaxisymmetric terms might contribute to the time-averaged field, although we suspect that none do. The present-day field has a large equatorial dipole component. Because

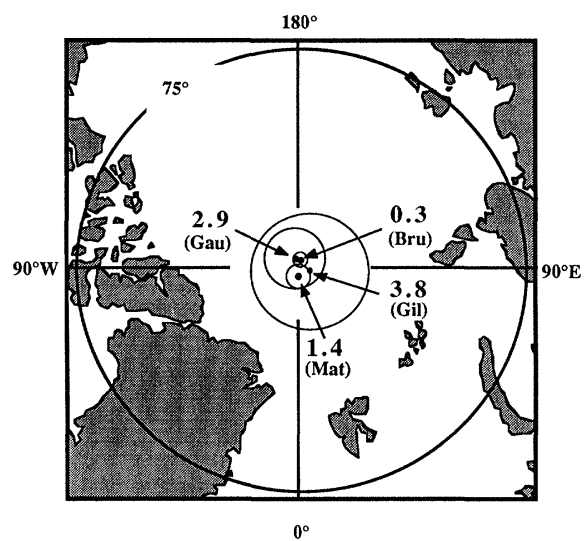

Fig. 6. Best fitting dipole axis position for Brunhes (Bru), Matuyama (Mat), Gauss (Gau), and Gilbert (Gil) chrons (solid points) with estimated circular standard deviations (open circles). Mean age of data included in each of the four groups is shown in millions of years.

secular variation is able to eliminate this gross axial asymmetry from the time-averaged field, it seems likely that, through time, higher order nonaxisymmetric components would be similarly removed.

We conclude that the time-averaged geomagnetic field is axially symmetric but that the strength of the NDF depends on polarity. This dependence must reflect a fundamental property of the time-averaged field, but it is inconsistent with the symmetry presumed of the basic physics of field generation (25). Merrill and McElhinney (4) have suggested that the NDF might be composed of a reversing component and a standing component that does not change when the main field reverses. The standing field would subtract from the reversing NDF during normal polarity intervals and add to it during reverse polarity intervals. Cast in these terms, our data would indicate a $4 \%$ reversing field (negative for normal dipole polarity; positive for reverse) and a $1 \%$ standing field (which is always positive). Although this standing NDF is only a small fraction of the time-averaged field, it might contribute substantially to the geomagnetic field during polarity transitions when the main dipole field collapses (25). The presence of a standing component during field transitions is consistent with zonal harmonic (26) models of field reversal and also is supported by transitional field observations. The standing field should cause transitional directions near the equator to go through steep, positive inclinations during the reversal process. Surveying published paleomagnetic records of equatorial transitional fields, we suggested that there are indeed steep, positive inclinations in many records from the equatorial zone (6).

How long might this standing field have persisted? And might it, like the main field, reverse polarity at times? Attempts have been made to estimate the magnitude of prePliocene NDFs (15); however, the extent of any polarity dependence is not known because the paleomagnetic data have not been separated by polarity. The source of the standing field identified in the Pliocene and Pleistocene is, in any case, uncertain. Merrill et al. considered thermo-electric currents at the core-mantle boundary as a possible source (25). Although they estimated that the likely lateral temperature variation would be too small to produce a substantial effect, more recent estimates (27) indicate that larger temperature variations do occur in the lower mantle, and some have loworder symmetry. Thus, large-scale thermoelectric currents may be able to generate a standing magnetic field (28). Alternatively, two independent modes, one associated with the main field and one with the standing field, may be an inherent part of the dynamo process.

\section{REFERENCES AND NOTES}

1. Wilson's examination of data in (2); R. L. Wilson, Geophys. J. R. Astron. Soc. 19, 417 (1970).

2. N. D. Opdyke and K. W. Henry, Earth Planet. Sci. Lett. 6, 139 (1969).

3. R. L. Wilson, Geophys. J. R. Astron. Soc. 28, 295 (1972).

4. R. T. Merrill and M. W. McElhinny, Rev. Geophys. Space Phys. 15, 309 (1977)

5. N. D. Opdyke, ibid. 10, 213 (1972)

6. D. A. Schneider and D. V. Kent, J. Geophys. Res., in press.

7. We included only cores having high-quality paleomagnetic records, with small scatter (standard deviation of inclination values are generally less than $15^{\circ}$ ) and well-determined magnetic polarity stratigraphy after alternating field demagnetization [see (6) for analysis techniques]. We did, however, allow considerable variation in the overall data quality; for example, generally poorer quality results from Atlantic cores were included in the data set. Our selection criteria were thus subjective but allowed us to balance the competing needs for high data quality and a good longitudinal site distribution.

8. P. L. McFadden and A. B. Reid, Geophys. J. R. Astron. Soc. 69, 307 (1982)

9. A. Cox, Rev. Geophys. Space Phys. 13, 35 (1975).

10. J. B. Minster and T. H. Jordan, J. Geophys. Res. 83, 5331 (1978)

11. The average $\Delta I$ for any particular core may be affected by systematic errors that could be of the same magnitude as the small NDF effects we are attempting to measure. Such errors may result from many sources including nonvertical penetration of the core barrel, perturbation by large, local magnetic fields (those associated with marine magnetic anomalies), and overprinting during handling and storage. We assume that such errors are random from core to core. Thus, computing an overall anomaly from the core averages give the best estimate of $\Delta I$ for each chron; the number of cores so averaged provides confidence limits.

12. R. F. King, Mon. Not. R. Astron. Soc. Geophys. Suppl. 7, 115 (1955)

13. A. Latham, Eos 69,546 (1988)

14. P. L. McFadden and M. W. McElhinny, J. Geomagn. Geoelectr. 34, 163 (1982)

15. D. H. Coupland and R. Van der Voo, J. Geophys. Res. 85, 3529 (1980)

16. R. A. Livermore, F. J. Vine, A. G. Smith, Geophys. J. R. Astron. Soc. 73, 153 (1983).

17. W. J. Morgan, in The Sea, C. Emiliani, Ed. (Wiley, New York, 1981), vol. 7, pp. 443-487.

18. J. A. Andrews, J. Geophys. Res. 90, 7737 (1985) 
19. V. Courtillot and J. Besse, Science 237, 1140 (1987).

20. C. G. A. Harrison and T. Lindh, Nature 300, 251 (1982)

21. R. A. Fisher, Proc. R. Soc. London, Ser. A 217, 295 (1953).

22. D. Gubbins, J. Geophys. Res. 93, 3413 (1988)

23. S. Lee, thesis, Australian National University, Canberra (1983).

24. D. A. Schneider and D. V. Kent, Geophys. Res. Lett. 13, 471 (1986).

25. R. T. Merrill, M. W. McElhinny, D. J. Stevenson, Phys. Earth Planet. Inter. 20, 75 (1979).

26. I. Williams and M. Fuller, J. Geophys. Res. 86, 11,657 (1981)

27. B. H. Hager, R. W. Clayton, M. A. Richards, R. P. Comer, A. M. Dziewonski, Nature 313, 541 (1985).

28. R. T. Merrill, Eos 68, 1210 (1987).

29. For Gauss and Gilbert groups, the uniform component has been set to the value determined by leastsquares analysis of Brunhes and Matuyama groups (see text). Fisher (21) precision parameter (kappa) and circular standard deviation (CSD) were estimated using a numerical simulation that adds Gaussian noise to inclination values represented in each data set. The variance of the Gaussian noise is set equal to the square of the root-mean-squares (rms) residual determined in the least-squares fit to the corre sponding data. These rms values range from $4.6^{\circ}$ (for the Brunhes chron) to $6.9^{\circ}$ (for the Gilbert chron). After adding noise, the best fitting dipole axis was recomputed by the least-squares method. This was repeated 1000 times and the results were averaged with standard Fisher statistics. From this average, we calculated the precision parameter and CSD and presume that these estimates roughly match those of the true population from which the best fitting dipole axis position was drawn.

30. We thank D. Johnson and L. Burckle for help determining biostratigraphic ages in many of the cores. We also thank R. Jarrard and $S$. Cande for constructive reviews of the manuscript. This work was supported by National Science Foundation Oceanography grants OCE-84-00805, OCE-8716672 , and core curatorial grant OCE-00232. Lamont-Doherty Geological Observatory contribution

4350 .

7 June 1988; accepted 22 August 1988

\title{
2,3,7,8-Tetrachlorodibenzo- $p$-dioxin Kills Immature Thymocytes by $\mathrm{Ca}^{2+}$-Mediated Endonuclease Activation
}

\author{
David J. McConkey, Pia Hartzell, Steven K. Duddy, \\ Helen Håkansson, STEN OrRenius*
}

Suspensions of thymocytes from young rats were incubated with 2,3,7,8-tetrachlorodibenzo- $p$-dioxin (TCDD), which resulted in a sustained increase in cytosolic free $\mathrm{Ca}^{2+}$ concentration followed by DNA fragmentation and loss of cell viability. Both the $\mathrm{Ca}^{2+}$ increase and DNA fragmentation were prevented in cells treated with the inhibitor of protein synthesis, cycloheximide, and DNA fragmentation and cell killing were not detected when cells were incubated in a "Ca ${ }^{2+}$-free" medium or pretreated with high concentrations of the calcium probe, quin-2 tetraacetoxymethyl ester. These results indicate that TCDD can kill immature thymocytes by initiating a suicide process similar to that previously described for glucocorticoid hormones.

G LUCOCORTICOID HORMONES, CYtotoxic $T$ lymphocytes, and natural killer cells all activate a process in target cells that is known as "apoptosis" or "programmed cell death" (1). Characteristic of this process is widespread chromatin condensation, which has been related to the stimulation of an endogenous endonuclease that cleaves host chromatin into oligonucleosome-length fragments (2). Recently we showed that an early, sustained increase in cytosolic $\mathrm{Ca}^{2+}$ concentration was critically involved in the activation of DNA fragmentation in glucocorticoid-treated thymocytes (3). A protein synthesized in response to glucocorticoid stimulation appeared to elevate the cytosolic $\mathrm{Ca}^{2+}$ level in thymocytes by facilitating the influx of extracellular $\mathrm{Ca}^{2+}$.

The compound 2,3,7,8-tetrachlorodibenzo- $p$-dioxin (TCDD) is the most potent

Department of Toxicology, Karolinska Institutet, Box 60400, S-104 01 Stockholm, Sweden.

*To whom correspondence should be addressed. of a group of halogenated, aromatic hydrocarbons that occur as industrial by-products and environmental contaminants. Despite extensive investigation, the mechanisms involved in TCDD-induced toxicity remain unknown. TCDD causes thymic atrophy in laboratory animals by depletion of the small, immature cortical cells of the thymus (4). This pattern of cell death resembles that observed in the thymus in response to glucocorticoid treatment (5). Glucocorticoids and TCDD also have overlapping or indistinguishable effects in many other target organs. For example, both are potent inducers of specific isozymes of cytochrome $\mathrm{P}$ 450 in the liver $(6)$, and both suppress B lymphocyte differentiation (7). In addition, the structural properties of the TCDD receptor are remarkably similar to those of the glucocorticoid receptor $(8)$. Since the purified glucocorticoid receptor binds selectively to regions of cloned DNA whose transcription is regulated by glucocorticoids in vivo, and the nucleotide sequences essential for receptor-DNA interaction are functional in vivo as hormone-dependent regulatory or transcriptional enhancer elements (9), the similar binding properties of the TCDD and glucocorticoid receptors could imply that they activate similar genetic targets, causing similar effects.

To determine whether TCDD could exert its influence on the thymus through endonuclease activation, we investigated the effects of TCDD on several parameters characteristic of apoptosis in immature thymocytes. Chromatin condensation is an early and characteristic morphological change occurring in apoptotic cells (1). Glucocorticoid hormones induce this morphology change in thymocytes soon after treatment. Chromatin condensation has been detected only in cells exhibiting extensive DNA fragmentation, and it is a property of apoptotic thymocytes that allows them to be separated from normal cells on the basis of bouyant density (10). Extensive plasma membrane and nuclear envelope blebbing is another morphological marker for programmed cell death that typically accompanies glucocorticoid-induced chromatin condensation (1). We found that after 1 hour of incubation the

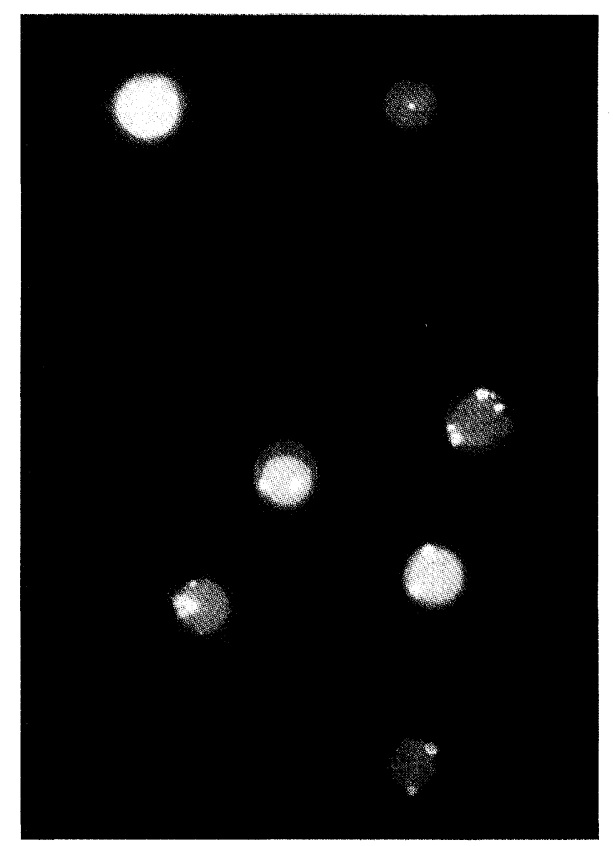

Fig. 1. Chromatin condensation in thymocytes exposed TCDD. Suspensions of thymocytes $\left(50 \times 10^{6}\right.$ per milliliter) from 3 -week-old (50- to $55-g)$ male Sprague-Dawley rats were incubated in rotating, round-bottomed flasks in Krebs-Hen-

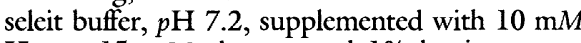
Hepes, $15 \mathrm{~m} M$ glucose, and $1 \%$ bovine serum albumin, at $37^{\circ} \mathrm{C}$ under an atmosphere of $95 \%$ $\mathrm{O}_{2}: 5 \% \mathrm{CO}_{2}$, in the presence of $10 \mathrm{nM} \mathrm{TCDD}$, dissolved in dimethyl sulfoxide, for 2 hours. Samples were then stained with acridine orange $(5 \mu \mathrm{l}$ of a solution containing $100 \mu \mathrm{g} / \mathrm{ml}$ plus $95 \mu \mathrm{l}$ of thymocyte suspension). Cells were then visualized with a fluorescence microscope. Magnification: $\times 1000$. 